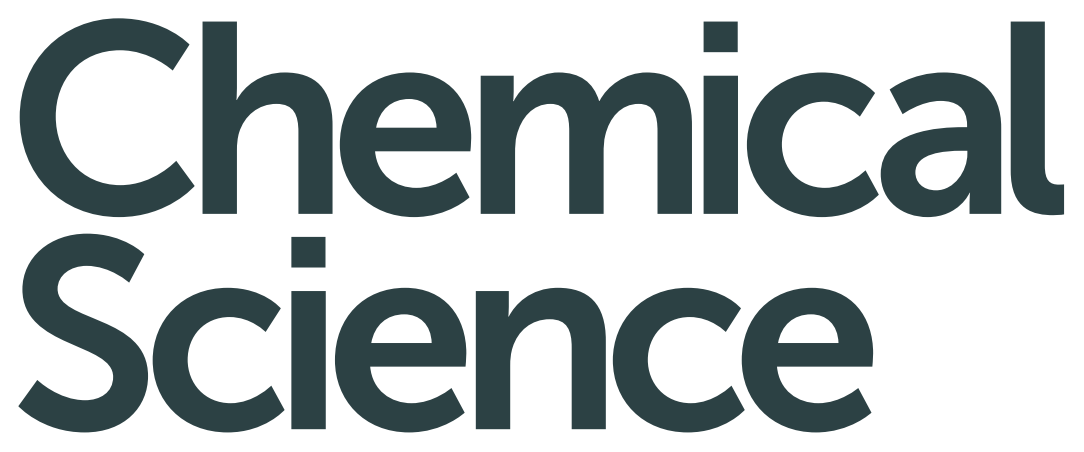

rsc.li/chemical-science

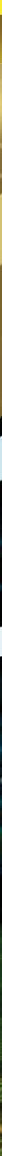

ISSN 2041-6539

EDGE ARTICLE

Zixuan Chen, Jun-Jie Zhu et al.

Dynamically imaging collision electrochemistry of single electrochemiluminescence nano-emitters 
Check for updates

Cite this: Chem. Sci., 2018, 9, 6167

๑ All publication charges for this article have been paid for by the Royal Society of Chemistry

Received 22nd May 2018

Accepted 29th June 2018

DOI: $10.1039 / \mathrm{c} 8 \mathrm{sc0} 02251 \mathrm{~h}$

rsc.li/chemical-science

\section{Dynamically imaging collision electrochemistry of single electrochemiluminescence nano-emitters $\dagger$}

\author{
Cheng Ma, Wanwan Wu, Lingling Li, Shaojun Wu, Jianrong Zhang, (D) Zixuan Chen (D) * \\ and Jun-Jie Zhu (D) *
}

\begin{abstract}
The quest for new techniques to measure single nanomaterials is a great impetus to research efforts to understand individual behaviours. Here, we develop an electrochemiluminescence (ECL) microscopy for visualization of stochastic collision electrochemistry of single nano-emitters without the interference of current and optical background. This design uses a water-immersion objective to capture the ECL emission of nanoparticles near the specular electrode surface for enhancing light collection efficiency. The approach enables us to trace the collision trajectory of multiple nanoparticles and spatially distinguish simultaneous collisions. Results reveal that collision types, frequencies and ECL intensities significantly depend on surface natures, particle concentrations, and diffusion fluxes. By recording successive collisions, we develop a "relay probe" sensing platform for long-term research. This imaging technique displays great potential for applications in single-particle electrochemical and analytical research.
\end{abstract}

The development of techniques on single nanoparticle measurements greatly promotes the fundamental research of single entities in nanoscale electrochemistry and applications in analysis, electrocatalysis, and biosensing. ${ }^{1-3}$ Among these techniques, ultramicroelectrodes (UMEs) provide a rapid and convenient stochastic collision strategy to characterize the nature of single nanoparticles, including electroactivity and size distribution. ${ }^{4-9}$ However, the detailed collision dynamics information obtained by traditional UMEs techniques is limited or can only be evaluated indirectly. ${ }^{10}$ As a result, the nanoparticles moving and simultaneously colliding on the surface of the electrode cannot be distinguished by potentiostats in a digital manner. Optical imaging techniques, such as fluorescence, surface plasmon resonance, and holographic microscopy, provide visualized approaches to describe the motion of single entities colliding with an electrode, enabling the dynamic study of individual electrochemical activity. ${ }^{11-15}$ But these optical techniques often require one or more extrinsic light sources, even high-power lasers, which disturb the electrochemical measurements by either inevitable optical background or local heat generation from the photothermal effect.

State Key Laboratory of Analytical Chemistry for Life Science, School of Chemistry and Chemical Engineering, Nanjing University, Nanjing 210023, P. R. China. E-mail: chenzixuan@nju.edu.cn; jjzhu@nju.edu.cn

$\dagger$ Electronic supplementary information (ESI) available: Additional experimental sections and more discussions (PDF). ECL imaging of four immobilized RuDSNs during a CV scanning (AVI). 3D motion trajectory during an elastic collision (AVI). Imaging simultaneous sticking collisions of three nanoparticles (AVI). Relay probes based on successive collision events (AVI). See DOI: $10.1039 / \mathrm{c} 8 \mathrm{sc} 02251 \mathrm{~h}$
Electrochemiluminescence (ECL) is an electrochemically triggered luminescence signal involving electron transfer reactions occurring at an electrode vicinity. ${ }^{16,17}$ Bard et al. demonstrate that ECL can provide an optical readout to study collision events of single entities without the interference of current background..$^{18-20}$ However, the non-imaging methods hardly attribute transient light signals to specific nanoparticles and it is difficult to attain the cyclic voltammogram of every nanoparticles. Recently, with benefits of the electron-multiplying charge-coupled device (EMCCD), ECL microscopy enables direct observation of individual immobilized entities with spatiotemporal resolution. ${ }^{21-23}$ Current ECL microscopy-based nanomaterials research focuses on the electrocatalytic amplification of bulk phase ECL luminophores on the surface of immobilized noble metal nanostructures, such as gold nanoparticles, gold nanowires, and gold-platinum Janus nanoparticles. ${ }^{24-26}$ Nevertheless, the background ECL emission from electrode substrates is still a significant issue that may disturb the electrochemical measurements. Moreover, the electrocatalytic reactions of metal materials are triggered only in the case of collision moment, so nanoparticles moving at an electrode vicinity and collision dynamics cannot be studied. Thus, developing a background-free ECL microscopy to image single nano-emitters at an electrode vicinity is critical for studying stochastic collision nanoelectrochemistry. However, techniques for mapping single ECL nano-emitters are still deficient up to now.

Here, we develop a single-nanoparticle ECL microscopy with co-localization of photoluminescence (PL) and scan electron microscopy (SEM) for directly observing the diversely collisional electrochemistry of nanoparticles. The well-known $\mathrm{Ru}(\mathrm{bpy})_{3}{ }^{2+}$ - 
doping silica nanoparticles (RuDSNs) are used here owing to its high ECL emission efficiency, ${ }^{27-29}$ though the ECL emission at single-nanoparticle level has not still been studied. We show the merits of the ECL microscopy for studying stochastic collision events and disclose how the nature of both the nanoparticle surface and electrode itself affects the dynamic collision behaviours. Moreover, based on continuous single-nanoparticle collisions, a unique "relay probe" concept is proposed to enable long-term observation and research. The developed ECL microscopy provides a zero background and visualization collision electrochemical technique at single-nanoparticle level.

\section{Results and discussion}

In order to observe single ECL nano-emitters, we develop a home-made upright microscope with a water-immersion objective $(\times 60, \mathrm{NA}=1.1)$ for higher light collecting efficiency and optical resolution (Fig. 1a). The RuDSNs are synthesized by the reverse microemulsion method according to the previous report (details in ESI $\dagger$ ). ${ }^{28}$ A three-electrode electrochemical cell with RuDSNs deposited on a specular glass carbon (GC) electrode is mounted under the objective with the electrolyte containing coreactant tri- $n$-propylamine (TPA). The potential is then cyclically scanned to excite ECL emission, which is collected by the objective to form ECL images (Fig. 1b). To accurately dissect the corresponding structures of these discrete ECL spots, we use SEM to characterize the detailed morphology of the same set of particles. The SEM co-localization images (Fig. 1c and $\mathrm{S} 3 \dagger$ ) provide direct evidence that the smallest ECL spots are from single nanoparticles $(\sim 62 \mathrm{~nm})$. Due to the restrict of optical diffraction limit, the nanoparticles close to each other are indistinguishable in ECL images, and thus produce bigger and brighter spots.

The co-localization approach reveals that the morphology of each particle significantly affects its ECL intensity. For example, the ECL intensity shows a pseudo-linear increment with the aggregates from monomer to hexamer (Fig. 1d), but noticeable error bars suggest more specific physical and chemical states of both particles and electrode, which also determine the ECL intensity. Moreover, the co-localization between ECL images and PL images (Fig. S1 and S2 $\dagger$ ) suggests that with the increase of doping amount of $\mathrm{Ru}(\mathrm{bpy})_{3}{ }^{2+}$ in a set of particles, the ECL intensity increases proportionally (Fig. 1e). Notice that some outliers suggest the doping heterogeneity in 3D siloxane nanomatrices. Avoiding ensemble averaging, the spatially resolved ECL microscopy with PL and SEM co-localization techniques not only enables us to identify the important features in the ECL emission of individual nanoparticles with high throughput, but also reveals the subtle relationship among the particle shape, doping amount, and ECL intensity.

Fig. 2 shows several ECL snapshots of four immobilized RuDSNs during a cyclic voltammetry scan (Movie S1 $\dagger$ ). The ECL spots appear following the oxidation of TPA (Fig. S4†) and reach the maximum brightness at $0.87 \mathrm{~V}$, corresponding to the "revisited" route involving both TPA cation radicals $\left(\mathrm{TPA}^{\cdot+}\right)$ and TPA free radicals (TPA ${ }^{\circ} .^{30}$ As the potential continues to scan beyond $0.87 \mathrm{~V}$, the ECL intensities of the four nanoparticles start to decrease, although the oxidation current increases and then reaches a peak at $1.05 \mathrm{~V}$. The contravention between the ECL emission intensity and the oxidation current is attributed
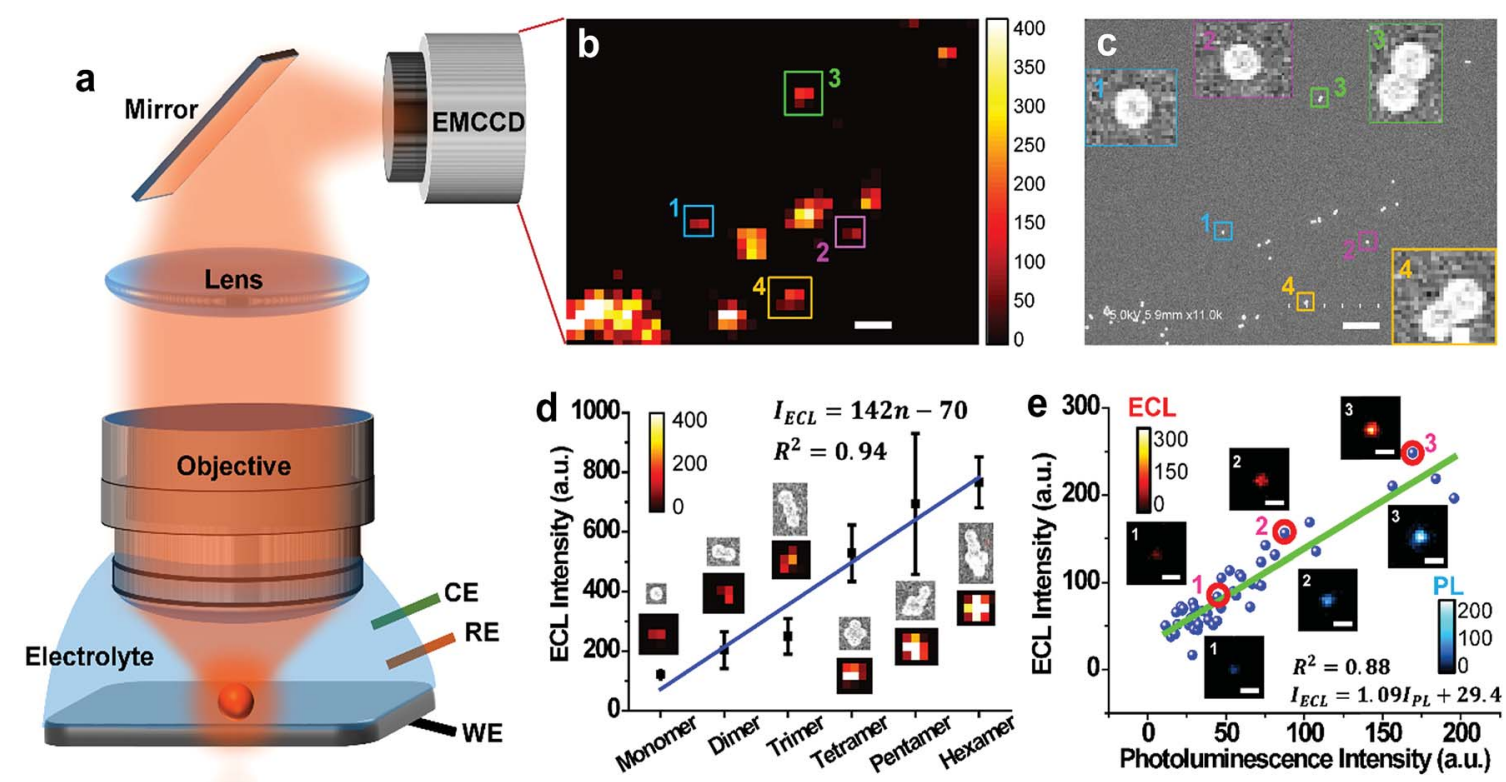

Fig. 1 (a) Schematic illustration of the ECL microscopy setup. (b) A zoom-in ECL image of the RuDSNs immobilized on the GC electrode in $100 \mathrm{mM}$ TPA solution. Scale bars (white), $1 \mu \mathrm{m}$. Exposure time: $1 \mathrm{~s}$. (c) The SEM image of the same set of particles as in (b). Insets show the detailed view of four individual RuDSNs numbered with 1-4. Scale bars (white), $1 \mu \mathrm{m}$. (d) Statistical ECL intensity from monomer to hexamer by the colocalization analyses between ECL images and SEM images. Error bars indicate the standard deviations $(n=3)$. Insets, representative ECL images of RuDSNs and corresponding SEM morphologies. (e) ECL intensity vs. PL intensity of the same set of RuDSNs. Insets, representative ECL images and PL images of RuDSNs numbered with 1-3. Scale bars (white), $2 \mu \mathrm{m}$. Exposure time of EMCCD are $1 \mathrm{~s}$ for both ECL and PL imaging 

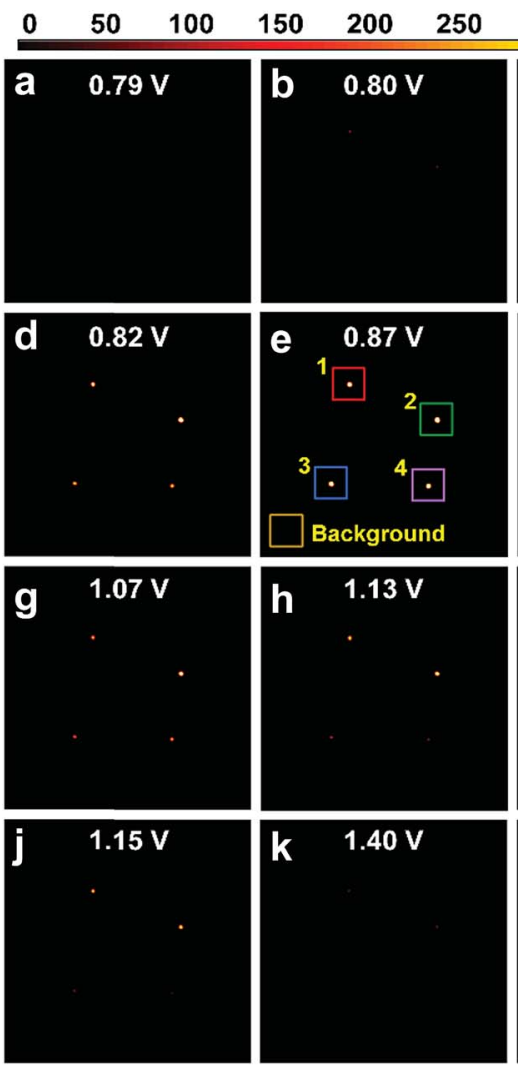
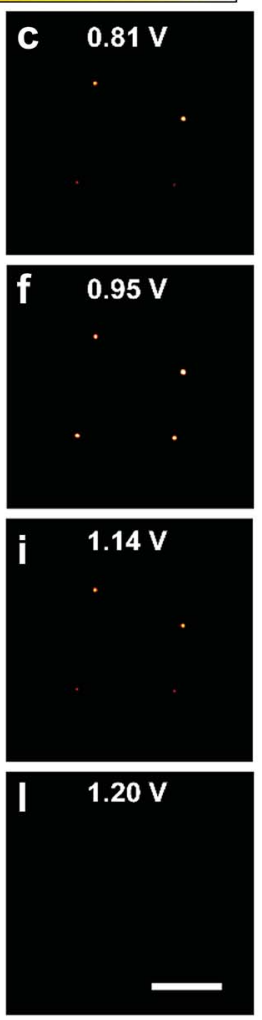

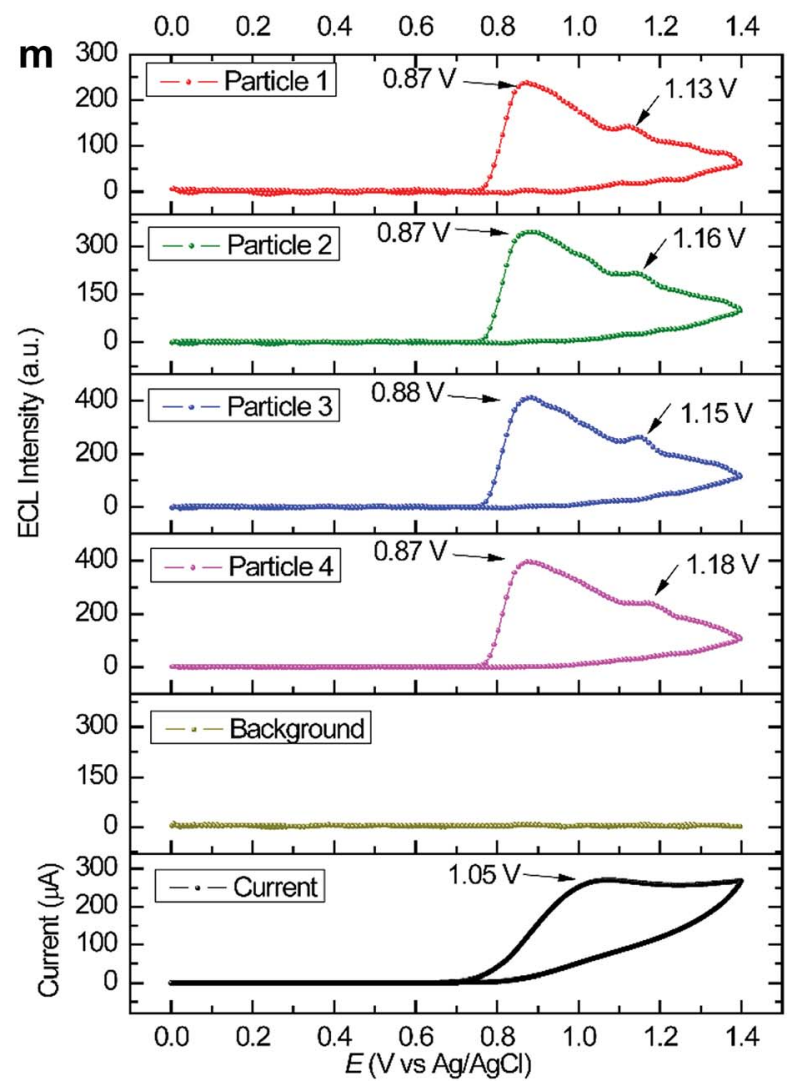

Fig. 2 (a-l) Successive ECL snapshots of four RuDSNs during a potential cycle. Scale bars (white), $30 \mu m$. (m) ECL intensity of four nanoparticles and blank area in (e) as a function of electrode potential and the corresponding CV curve of the GC electrode (black line) recorded by the potentiostat. The potential was cyclically scanned from 0 to $1.4 \mathrm{~V}$ at a scan rate of $20 \mathrm{mV} \mathrm{s}^{-1}$ in $200 \mathrm{mM}$ PBS buffer containing $100 \mathrm{mM}$ TPA. The exposure time of EMCCD was $500 \mathrm{~ms}$

to direct anodic oxidation of TPA ${ }^{*}$ at the electrode interface..$^{31,32}$ It is noteworthy that we observe another weak ECL peak around $1.16 \mathrm{~V}$, derived from the direct oxidation of the $\mathrm{Ru}(\mathrm{bpy})_{3}{ }^{2+}$ molecules inside the RuDSNs (Fig. S5 $\dagger$ ). ${ }^{33}$ Thus, the electron tunnelling/hopping mechanism is experimentally confirmed at single-nanoparticle level. The ECL-potential curve of single nanoparticles can accurately reveal specific electrochemical reaction without the interference from the charge-discharge current, side reactions and water decomposition reaction, which are inevitable in traditional $\mathrm{CV}$ measurements by macroelectrodes. In addition, as illustrated in Fig. $2 \mathrm{~m}$, the ECLpotential curve shows a zero background signal from the blank electrode substrate during the entire ECL process, which demonstrates the high accuracy and sensitivity for imaging local electrochemical reactions by our ECL microscopy. Fig. S6† shows the ECL-potential curves of individual RuDSNs at different electrode surfaces. The obvious differences in both the ECL peak potential and the ECL intensity suggest the ECL reactions kinetics significantly depends on the electrode material and interfacial properties (details in ESI $\dagger$ ).

To gain more insights into the way how the particle size and the distance from electrode surface affect the ECL emission efficiency, we synthesize $\mathrm{Ru}(\mathrm{bpy})_{3}{ }^{2+}$-coating silica particles (RuCSPs) with three different sizes (details in ESI $\dagger$ ) in order to establish the theoretical model, because ECL reactions only occur on the particle surface. These three RuCSPs are imaged by the PL and ECL co-localization technique. TEM images (Fig. 3ac) show that the three RuCSPs have well-defined spherical shapes with average diameters of $90 \mathrm{~nm}, 420 \mathrm{~nm}$ and $1.5 \mu \mathrm{m}$, respectively. The PL intensity (Fig. 3d-f) are proportional to the superficial areas of themselves, suggesting the same surface density of $\mathrm{Ru}(\mathrm{bpy})_{3}{ }^{2+}$ in different sized RuCSPs. Nevertheless, the increase rates of the ECL intensity are much lower relative to that of their superficial areas and PL intensity (Fig. 3g-i and m). To understand the difference, the three-dimensional ECL patterns of each RuCSP are simulated by a previously reported method (details in ESI $\dagger$ ). ${ }^{34}$ The ECL patterns from the side-view show exponential decreasing brightness from the bottom toward the top (Fig. 3j-l). The decreasing ECL efficiency is mainly attributed to two factors. The first is the electron tunnelling/hopping and lateral charge propagation of $\mathrm{Ru}(\mathrm{bpy})_{3}{ }^{2+}$, which depend on the distance from the electrode surface. The other is decreasing concentration of chemicallyunstable TPA $^{\cdot+}$ with diffusing from the electrode surface, which produce ECL emission by the "revisited" mechanism. Thus, the ECL efficiency highly depends on the distance from the electrode surface (Fig. 3n), leading to merely a tiny proportion of $\mathrm{Ru}(\mathrm{bpy})_{3}{ }^{2+}$ involved in ECL reactions. On the 

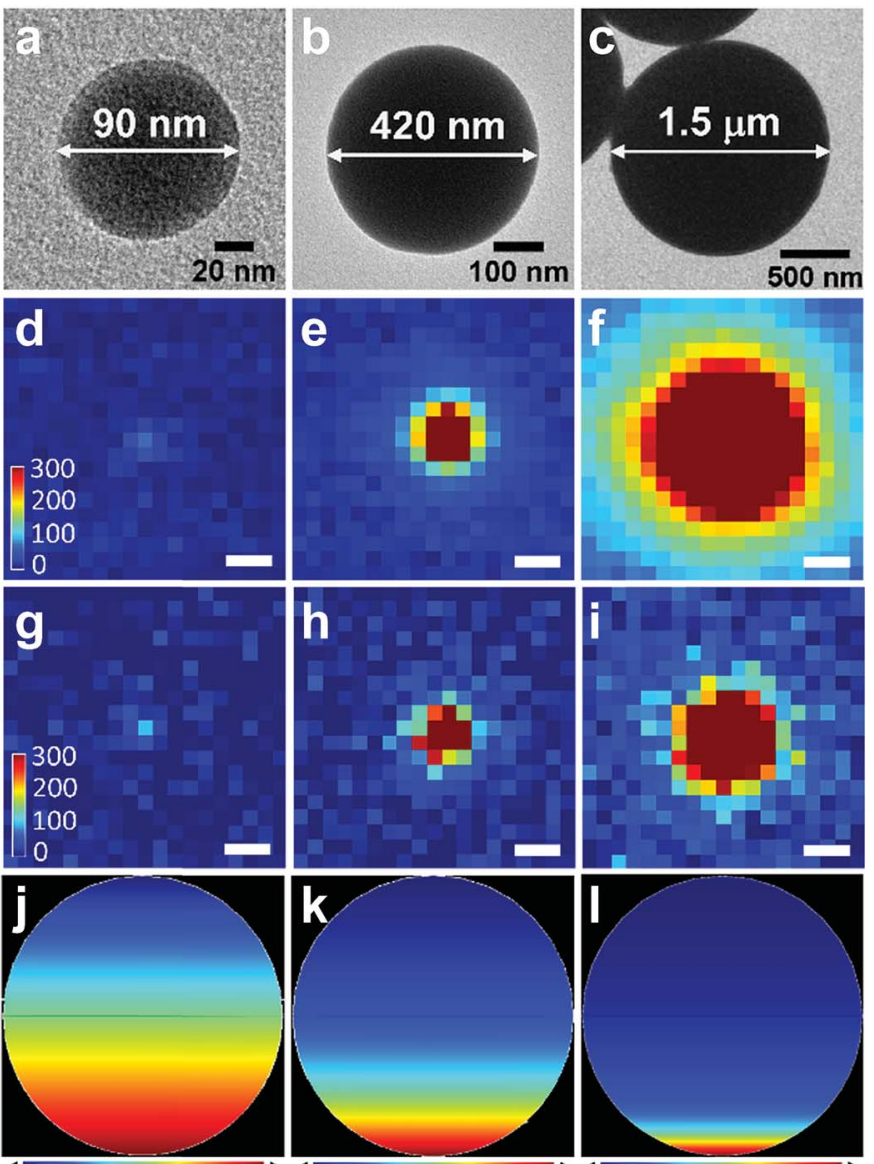

$4.24 \times 10^{-6} \quad 5.67 \times 10^{-6} 9.42 \times 10^{-7} \quad 7.8 \times 10^{-6} 1.68 \times 10^{-7} \quad 9.46 \times 10^{-6}$

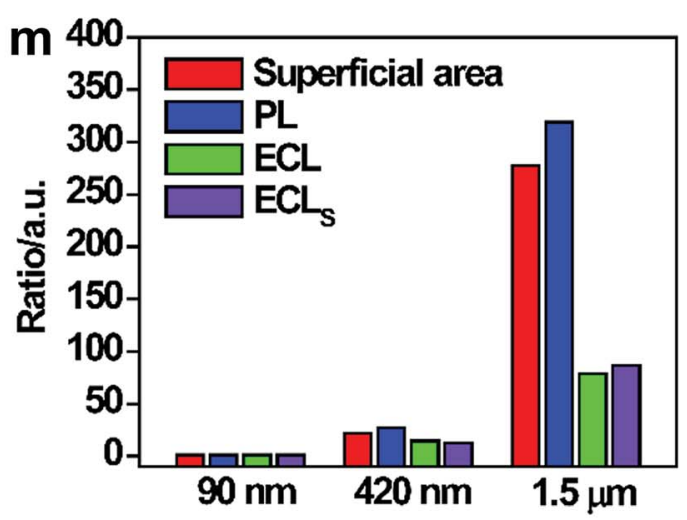

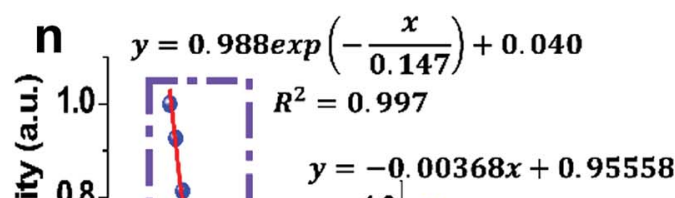

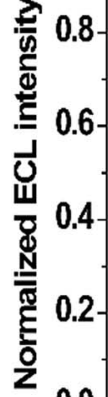

0.0 .

$\begin{array}{lllllllllll}-0.2 & 0.0 & 0.2 & 0.4 & 0.6 & 0.8 & 1.0 & 1.2 & 1.4 & 1.6\end{array}$ Distance from electrode surface $(\mu \mathrm{m})$

Fig. 3 TEM ( $a-c), P L(d-f)$, and ECL $(g-i)$ images and side-view images of simulated ECL patterns (j-l) of single $90 \mathrm{~nm}, 420 \mathrm{~nm}$, and $1.5 \mu \mathrm{m}$ $\mathrm{Ru}(\mathrm{bpy})_{3}{ }^{2+}$-coating silica particles (RuCSPs), respectively. Scale bars (white), $1 \mu \mathrm{m}$. Exposure time of both ECL images and PL images are $1 \mathrm{~s}$. (m) Ratios of three RuCSPs in terms of superficial area, PL intensity, ECL intensity and simulated ECL intensity $\left(E C L_{s}\right)$, which are normalized by 90 nm RuCSP. (n) The relationship between the normalized ECL intensity and the distance from the electrode surface. The blue dots and the red line represent the ECL intensity at different distances and the fitting curve, respectively. Inset shows a zoom-in view of the ECL intensity within $200 \mathrm{~nm}$ distance from electrode surface.

other hand, distance-dependence of the ECL efficiency enables us to measure the distance from the position of mobile nanoparticles to the electrode surface, given that the ECL intensity shows high sensitivity and a linear relation within $\sim 200 \mathrm{~nm}$ from the electrode (inset in Fig. 3n). Fig. S7† shows the ECL emission of RuDSNs even on the upper surface of single HeLa cell, which serves as a "barrier" between the particles and the electrode. Thus, the ECL emission far from the electrode enables us to image mobile RuDSNs in the vicinity of electrodes and further study the collision dynamics (see below).

Single nanoparticle collisions are recorded on injecting RuDSNs into the electrolyte containing the coreactant TPA and the bare GC electrode (applied potential of $1.4 \mathrm{~V}$ ). Initially, no ECL signal is observed on the electrode surface. But shortly afterwards, several discrete ECL spots appear successively in different regions, demonstrating direct visualization of collision electrochemistry. These discrete ECL signals fall into two major categories: "spike" and "staircase" (Fig. 4a and b), suggesting the elastic and sticking collision, respectively. Oftentimes nanoparticles find way back to the electrode after an elastic collision to generate continuous collision events (Fig. S8†). A bunch of collisions in a local region imply the random walk of single nanoparticles in the vicinity of electrode surface. ${ }^{6,35}$ Interestingly, during the collision process, the variation of the ECL intensity is accompanied by horizontal motions of the spot (Fig. 4c), indicating Brownian motion of single RuDSN in all direction. By fitting its diffraction-limited emission (Fig. S9 $\dagger$ ) and solving the intensity-distance equation in Fig. 3n, we disclose the three-dimensional spatial position of the RuDSN during the collision process (Fig. 4d and Movie S2 $\dagger$ ). So far, the temporal resolution of ECL microscopy is limited to the ECL intensity of single ECL nano-emitters. Therefore, the temporal resolution used in this work $(0.2 \mathrm{~s})$ is quite fast in the current research. Because of the rapid motion of the nanoparticle, the detailed motion trajectory and the instantaneous kinetic velocity of the RuDSN cannot be accurately calculated and described in this work. Nevertheless, every ECL spot represents the most probable position of the nanoparticle at that time, so the ECL microscopy provides the visualization of the spatial motion information during the collision 

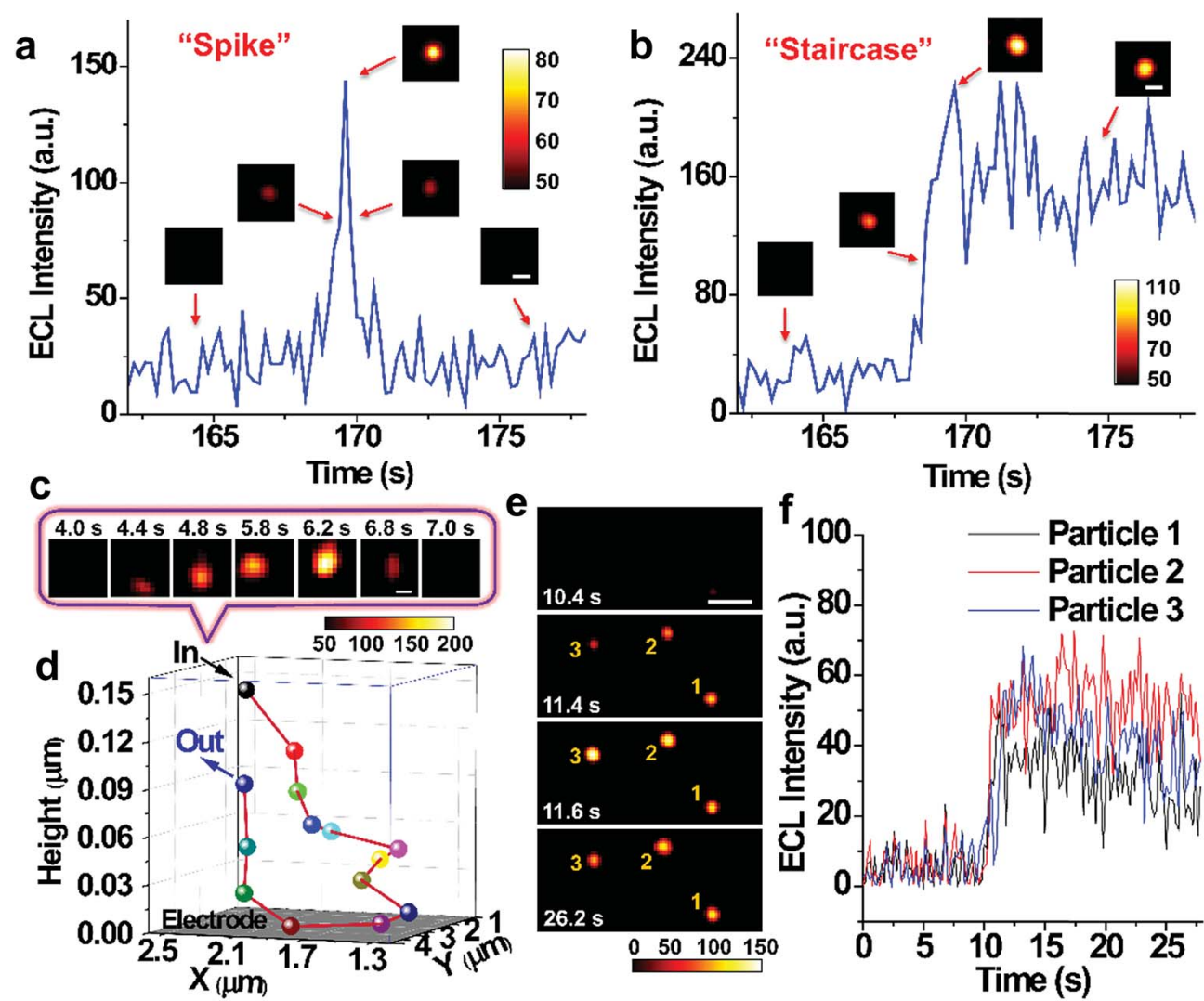

Fig. 4 Single ECL spike (a) and staircase (b) signals during the collisions process. Insets, ECL snapshots of individual nanoparticles during a typical collision process. Constant potential: $1.4 \mathrm{~V}$. Scale bars (white), $2 \mu \mathrm{m}$. Exposure time: $0.2 \mathrm{~s}$. (c) ECL snapshots of individual nanoparticle during an elastic collision with Brownian motion. Scale bars (white), $1 \mu \mathrm{m}$. Exposure time: $0.2 \mathrm{~s}$. (d) Three-dimensional collision trajectory of single nanoparticle in (c). (e) ECL snapshots of three RuDSNs colliding with the electrode simultaneously. Scale bars (white), $5 \mu \mathrm{m}$. Exposure time: $0.2 \mathrm{~s}$. (f) ECL intensity vs. time curves of three RuDSNs in (e).

electrochemistry process. In the future, the temporal resolution of ECL microscopy can be improved by either developing novel ECL nano-emitters with higher emission efficiency or optimize the microscope configurations, and the more detailed dynamic pictures of the collision process can be achieved.

The large view field $(164 \mu \mathrm{m} \times 164 \mu \mathrm{m})$ enables us to observe multiple collision events simultaneously. For example, Fig. 4e and $\mathrm{f}$ and Movie $\mathrm{S} 3 \uparrow$ show no ECL signal on the electrode surface in the first $10.4 \mathrm{~s}$. But in the following $1.2 \mathrm{~s}$, three weak ECL spots appear in the blank and gradually brighten, suggesting three particles approaching the electrode surface. Finally, three spots reach the maximum brightness at the same time, indicating three simultaneous sticking collisions. Note that simultaneous collisions of multiple particles lead to an overlapped transient ECL or current signal with traditional UMEs techniques, ${ }^{19}$ which highlights the value of spatial resolution provided by the ECL microscopy in this work.

The probabilities of sticking collisions are investigated with different electrode surface modifications and nanoparticle capping agents (Fig. 5a). For instance, coating RuDSNs with bovine serum albumin (BSA) results in about a five times lower sticking probability relative to bare RuDSNs, attributed to the inhibited nonspecific adsorption by the steric hindrance of BSA (Fig. S10†). On the other hand, the sticking probability increases when blocking the bare GC electrode with poly (sodium 4-styrenesulfonate) (PSS), which offers abundant negative charges to irreversibly adsorb positively charged RuDSNs by electrostatic interaction. More detailed descriptions are in ESI. $\dagger$ These results elucidate that the probability of sticking collisions is a function of the interaction between the nanoparticles and electrode, and very sensitive to the steric hindrance and surface charges.

An interesting discovery is that RuDSNs tend to collide with greater intensity and frequency near the edge of the GC electrode than at the interior zone (Fig. S11 $\dagger$ ). This result demonstrates higher diffusive flux of both the RuDSNs and coreactant TPA to the edges favors the ECL intensity and collision frequency. The collision frequency $\left(f_{\mathrm{p}}\right)$ of the overall electrode surface can be estimated by the steady-state diffusion-limited model $\left(f_{\mathrm{p}} \approx 4 D^{\mathrm{p}} c^{\mathrm{p}} r^{\mathrm{d}}\right.$, where $D^{\mathrm{p}}$ is the diffusion coefficient of particles, $c^{\mathrm{p}}$ is the concentration of the RuDSNs, and $r^{\mathrm{d}}$ is the radius of the GC electrode). ${ }^{35}$ As shown in Fig. $5 \mathrm{~b}$, the calculated values are slightly less than the experimental values, which are obtained from the Fig. S11e $\uparrow$ by the integrated method. The deviations are probably derived from the continuous collisions of some RuDSNs according to the random walk behaviour (Fig. S8 $\dagger$ ), which is ignored in the diffusion-limited theoretical model. 

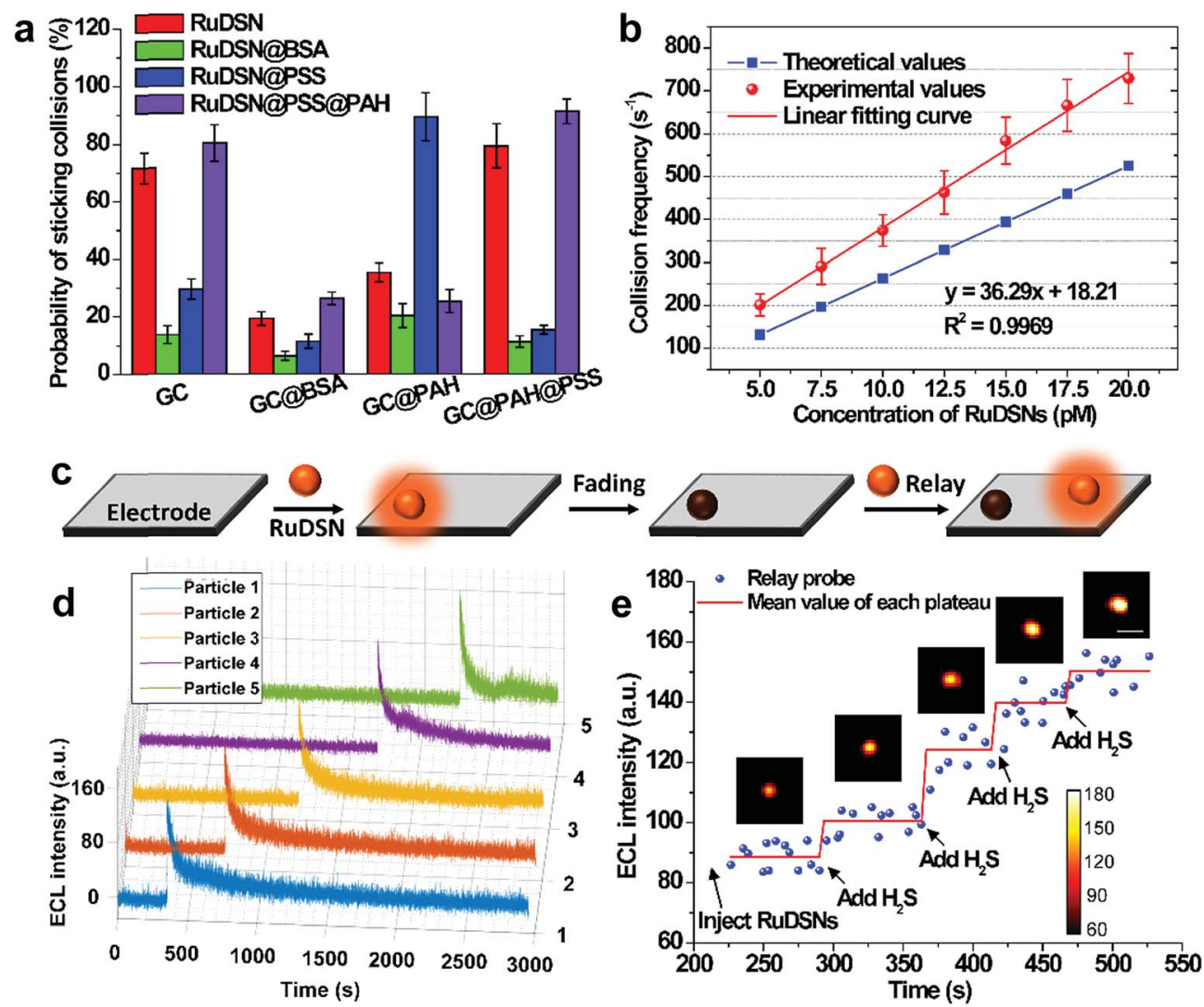

Fig. 5 (a) Probabilities of sticking collisions with different electrode surface modifications and nanoparticle capping agents. (b) Theoretical and experimental collision frequencies of the overall electrode surface with different particle concentrations. (c) "Relay probe" operation procedure by recording successive collision events from different nanoparticles. (d) ECL intensity vs. time curves of five nanoparticles sticking to electrode at different times. (e) "Relay probe" for the real-time analysis of $\mathrm{H}_{2} \mathrm{~S}$ concentration in a long-term research. Every blue dot represents the collisional $E C L$ intensity of each relay probe. Insets: Representative ECL snapshots of relay probes in each plateau. Scale bars (white), $3 \mu \mathrm{m}$. Exposure time: $0.2 \mathrm{~s}$.

Statistics indicate that the collision events from different nanoparticles have similar ECL intensity (Fig. S12†). However, concerning single RuDSN, once it sticks to the electrode, its ECL intensity decays exponentially with the time elapse (half-life time $=29.5 \pm 4.8 \mathrm{~s}$ ) (Fig. $5 \mathrm{~d}$ ). The dissolution or passivation of RuDSNs seems to be impossible because of the inert electrochemical property of the silica matrix. Nevertheless, the obvious decline of the PL intensity of RuDSNs after the ECL reactions (Fig. S13†) suggests that the ECL decay is attributed to the depletion of the $\mathrm{Ru}(\mathrm{bpy})_{3}{ }^{2+}$ molecules inside nanoparticles during the ECL reactions. As a result, only single nanoparticle hardly serves as the nanoprobe for long life-time research. To address this problem, we use a strategy called "relay probe" ${ }^{36}$ to record the collisional ECL emission of different nanoparticles with time trajectory. As shown in Fig. 5c, once prior RuDSNs stick to the electrode and become deactivation, immediately following RuDSNs work instead. To demonstrate this concept, we employ a $\mathrm{H}_{2} \mathrm{~S}$-responsive dye (CouMC) to quench the ECL emission of RuDSNs (Fig. S15†). ${ }^{37}$ In the presence of CouMC, the collisional ECL intensities from different particles are all very weak. But when successively adding $\mathrm{H}_{2} \mathrm{~S}$ into the solution, the intensities of freshly adherent RuDSNs quickly increase and reach plateaus (Fig. 5e and Movie S4†). The average collisional
ECL intensity in each plateau is nearly proportional to the concentration of $\mathrm{H}_{2} \mathrm{~S}$ (Fig. S16 $\dagger$ ). Although every RuDSN sticking to electrode shows rapidly decay with the time elapse, the "relay probe" strategy successfully avoids the deficiency by statistically analyzing successive collision events. This strategy provides a collision-based sensing platform for long life-time research.

\section{Conclusions}

In summary, the ECL microscopy provides a visualization method to dynamically study the stochastic collision electrochemistry of single nano-emitters without the interference of current and optical background. Co-localization analyses among ECL, PL, and SEM images reveal that the ECL emission is closely related to morphologies, doping amounts, particle sizes, and distances from the electrode surface, which is further confirmed by the theoretical simulations. By using this technique, we successfully trace the three dimensional collision trajectory and spatially distinguish simultaneous collision events of multiple nanoparticles, which give access to kinetic study of nanoparticles with high-throughput. Furthermore, we disclose the single-nanoparticle collision behaviours, including 
collision types, frequencies, and collisional ECL intensities are significantly correlated with interfacial properties, particle concentrations, and diffusion fluxes. By recording large numbers of collision events with time elapse, we develop a "relay probe" method to enable long-term observation and research. As an electrochemically triggered luminescence imaging technique, the background-free and spatiotemporally resolved ECL microscopy displays great potential for applications in single-particle electrochemical and analytical research.

\section{Experimental section}

\section{ECL with co-localization of PL microscopy set-up}

As illustrated in Fig. S1, $\uparrow$ the ECL imaging was performed on a homemade upright configuration microscope, which was established based on a weak light collecting microscope integrating the bright field, PL and ECL imaging. A water immersion objective (Olympus LUMFLN $\times 60, \mathrm{NA}=1.10$ ) was used for collecting ECL emission. According to $R=0.61 \lambda / \mathrm{NA}(R$ is the optical resolution; $\lambda$ is the emission wavelength; NA is the numerical aperture of the objective), the optical resolution of the microscope is $\sim 300 \mathrm{~nm}$. An EMCCD camera (Photometrics, Evolve 512 Delta, $512 \times 512$ pixels) was used for bright field, PL and ECL imaging. A potentiostat (CHI 660D electrochemical workstation) was used to control the electrode potential with an $\mathrm{Ag} / \mathrm{AgCl}$ (saturated $\mathrm{KCl}$ ) and a Pt wire as the reference electrode and the counter electrode, respectively. The EMCCD was triggered and synchronized by the potentiostat. PL imaging was also performed on this microscope by using a mercury lamp (Nikon Intensilight C-HGFI), fluorescent filters and a dichroic mirror (Fig. S1b†े). Other optical elements and optomechanical components were purchased from Thorlabs, Inc.

\section{Single-nanoparticle ECL imaging with co-localization of SEM}

Prior to use, a dismountable GC electrodes ( $3 \mathrm{~mm}$ in diameter) was polished sequentially with 0.3 and $0.05 \mu \mathrm{m}$ alumina slurry on an abrasive cloth, followed by ultrasonic cleaning with ethanol and ultrapure water thoroughly. Then the electrode was allowed to dry under a $\mathrm{N}_{2}$ flow. For co-localization analysis with SEM, the GC electrode surface was scratched by a knife to form a special pattern. Then RuDSNs (62 $\mathrm{nm}$ ) dispersed in ethanol solution was dropped $(1 \mu \mathrm{L})$ on the pretreated GC electrode surface. After drying in air, a three-electrode electrochemical cell made by PDMS with GC electrode working electrode (an Ag/ $\mathrm{AgCl}$ as reference electrode and a Pt wire as the counter electrode) was mounted on a high numerical aperture water immersion objective. Subsequently, $1 \mathrm{~mL}$ PBS $(10 \times, \mathrm{pH}=7.0)$ buffer containing $100 \mathrm{mM}$ TPA as the coreactant was added in the electrochemical cell. After covering the microscope with a light-tight box, the ECL signal was initiated and controlled by switching an electrode voltage, and then was collected by the objective and imaged on the EMCCD. Then, a $\mathrm{N}_{2}$ flow was used to blow the electrolyte solution off the electrode surface, followed by the disassembly of the GC electrode head for SEM experiment.

\section{Imaging stochastic collision nanoelectrochemistry}

To perform the collision experiment, the bare GC electrode was mounted under the water immersion objective with an $\mathrm{Ag} / \mathrm{AgCl}$ as reference electrode and a Pt wire as the counter electrode. In order to remove the aggregates of RuDSNs and obtain the monodisperse RuDSNs, the RuDSNs sample was filtered by 0.22 $\mu \mathrm{m}$ ultrafiltration membranes and then centrifuged at $5000 \mathrm{rpm}$ for $5 \mathrm{~min}$. The transparent supernatant containing monodisperse RuDSNs was collected for the following collision experiments. The electrolyte was $200 \mathrm{mM}$ PBS buffer $(\mathrm{pH}=7.0)$ containing $100 \mathrm{mM}$ TPA. The EMCCD was triggered and synchronized by the potentiostat when a constant voltage $(1.4 \mathrm{~V})$ was imposed on the GC electrode. After the current reached stability, the RuDSNs was injected into the electrolyte, and then the ECL signal from transient collisions was recorded by the EMCCD (exposure time: $0.2 \mathrm{~s}$ ). All data processing was performed with Matlab and ImageJ software. The collisional ECL intensity meant the maximum ECL intensity of transient collision with time trajectory in all collision experiments. The distributions of statistic collision intensities, frequencies, and types were recorded in the center of CCD-view $(100 \mu \mathrm{m} \times 100$ $\mu \mathrm{m})$.

\section{COMSOL simulation for sub-particle ECL pattern analysis}

To understand the sub-particle ECL patterns of RuCSPs $(90 \mathrm{~nm}$, $420 \mathrm{~nm}$ and $1.5 \mu \mathrm{m}$ ), the finite element analyses were conducted by using COMSOL Multiphysics software (version 5.2). The component with $2 \mathrm{D}$ axisymmetric geometry was used in this model. The kinetic and thermodynamic parameters were obtained according to the values suggested by Miao and Sojic. ${ }^{30,34}$ The proposed model involved a large amount of diffusion of the species, charge transfer reactions at the electrode surface, and chemical reactions at the particle surface and in homogeneous solution. The Butler-Volmer formalism was used to calculate the kinetics of the charge transfer reactions. The oxidation rates were very fast so that the oxidation processes were controlled by the species diffusion. Fick equations were used to describe the flux rates of species in the electrode vicinity.

\section{Conflicts of interest}

The authors declare no competing financial interest.

\section{Acknowledgements}

This research is supported by the National Natural Science Foundation of China (Grants No. 21335004, 21427807 and 21605081), the Natural Science Foundation of Jiangsu Province (Grants No. BK20160638), China Postdoctoral Science Foundation (Grants No. 2016M590434, and 2017T100345), and the International Cooperation Foundation from Ministry of Science and Technology (2016YFE0130100). We thank Dr Jianbin Pan for helping build the triggering and synchronization systems between the ECL microscope and the potentiostat. We thank Dr Zengqiang Wu for helping perform the numerical simulation. 


\section{References}

1 R. W. Murray, Nanoelectrochemistry: Metal Nanoparticles, Nanoelectrodes, and Nanopores, Chem. Rev., 2008, 108, 2688-2720.

2 J. B. Sambur, T. Y. Chen, E. Choudhary, G. Chen, E. J. Nissen, E. M. Thomas, N. Zou and P. Chen, Sub-Particle Reaction and Photocurrent Mapping to Optimize Catalyst-Modified Photoanodes, Nature, 2016, 530, 77-80.

$3 \mathrm{~W}$. Cheng and R. G. Compton, Quantifying the Electrocatalytic Turnover of Vitamin B12-Mediated Dehalogenation on Single Soft Nanoparticles, Angew. Chem., Int. Ed., 2016, 55, 2545-2549.

4 R. Dasari, K. Tai, D. A. Robinson and K. J. Stevenson, Electrochemical Monitoring of Single Nanoparticle Collisions at Mercury-Modified Platinum Ultramicroelectrodes, ACS Nano, 2014, 8, 4539-4546.

5 Y. Y. Peng, R. C. Qian, M. E. Hafez and Y. T. Long, Stochastic Collision Nanoelectrochemistry: A Review of Recent Developments, ChemElectroChem, 2017, 4, 977-985.

6 D. A. Robinson, Y. Liu, M. A. Edwards, N. J. Vitti, S. M. Oja, B. Zhang and H. S. White, Collision Dynamics During the Electrooxidation of Individual Silver Nanoparticles, J. Am. Chem. Soc., 2017, 139, 16923-16931.

7 Z. Guo, S. J. Percival and B. Zhang, Chemically Resolved Transient Collision Events of Single Electrocatalytic Nanoparticles, J. Am. Chem. Soc., 2014, 136, 8879-8882.

8 D. A. Robinson, J. J. Yoo, A. D. Castañeda, B. Gu, R. Dasari, R. M. Crooks and K. J. Stevenson, Increasing the Collision Rate of Particle Impact Electroanalysis with Magnetically Guided Pt-Decorated Iron Oxide Nanoparticles, ACS Nano, 2015, 9, 7583-7595.

9 E. J. E. Stuart, K. Tschulik, C. Batchelor-McAuley and R. G. Compton, Electrochemical Observation of Single Collision Events: Fullerene Nanoparticles, ACS Nano, 2014, 8, 7648-7654.

10 T. J. Anderson and B. Zhang, Single-Nanoparticle Electrochemistry through Immobilization and Collision, Acc. Chem. Res., 2016, 49, 2625-2631.

11 S. E. Fosdick, M. J. Anderson, E. G. Nettleton and R. M. Crooks, Correlated Electrochemical and Optical Tracking of Discrete Collision Events, J. Am. Chem. Soc., 2013, 135, 5994-5997.

12 R. Hao, Y. Fan and B. Zhang, Imaging Dynamic Collision and Oxidation of Single Silver Nanoparticles at the Electrode/Solution Interface, J. Am. Chem. Soc., 2017, 139, 12274-12282.

13 Y. Fang, W. Wang, X. Wo, Y. Luo, S. Yin, Y. Wang, X. Shan and N. Tao, Plasmonic Imaging of Electrochemical Oxidation of Single Nanoparticles, J. Am. Chem. Soc., 2014, 136, 12584-12587.

14 A. N. Patel, A. Martinez-Marrades, V. Brasiliense, D. Koshelev, M. Besbes, R. Kuszelewicz, C. Combellas, G. Tessier and F. Kanoufi, Deciphering the Elementary Steps of Transport-Reaction Processes at Individual $\mathrm{Ag}$
Nanoparticles by 3d Superlocalization Microscopy, Nano Lett., 2015, 15, 6454-6463.

15 V. Brasiliense, A. N. Patel, A. Martinez-Marrades, J. Shi, Y. Chen, C. Combellas, G. Tessier and F. Kanoufi, Correlated Electrochemical and Optical Detection Reveals the Chemical Reactivity of Individual Silver Nanoparticles, J. Am. Chem. Soc., 2016, 138, 3478-3483.

16 D. M. Hercules, Chemiluminescence Resulting from Electrochemically Generated Species, Science, 1964, 145, 808-809.

17 Z. F. Ding, B. M. Quinn, S. K. Haram, L. E. Pell, B. A. Korgel and A. J. Bard, Electrochemistry and Electrogenerated Chemiluminescence from Silicon Nanocrystal Quantum Dots, Science, 2002, 296, 1293-1297.

18 F. R. Fan and A. J. Bard, Observing Single Nanoparticle Collisions by Electrogenerated Chemiluminescence Amplification, Nano Lett., 2008, 8, 1746-1749.

19 J. E. Dick, C. Renault, B. K. Kim and A. J. Bard, Simultaneous Detection of Single Attoliter Droplet Collisions by Electrochemical and Electrogenerated Chemiluminescent Responses, Angew. Chem., Int. Ed., 2014, 53, 11859-11862.

20 F. R. F. Fan, S. Park, Y. Zhu, R. S. Ruoff and A. J. Bard, Electrogenerated Chemiluminescence of Partially Oxidized Highly Oriented Pyrolytic Graphite Surfaces and of Graphene Oxide Nanoparticles, J. Am. Chem. Soc., 2009, 131, 937-939.

21 Y. Chang, R. E. Palacios, F. R. Fan, A. J. Bard and P. F. Barbara, Electrogenerated Chemiluminescence of Single Conjugated Polymer Nanoparticles, J. Am. Chem. Soc., 2008, 130, 8906-8907.

22 F. Deiss, C. N. LaFratta, M. Symer, T. M. Blicharz, N. Sojic and D. R. Walt, Multiplexed Sandwich Immunoassays Using Electrochemiluminescence Imaging Resolved at the Single Bead Level, J. Am. Chem. Soc., 2009, 131, 6088-6089.

23 G. Valenti, S. Scarabino, B. Goudeau, A. Lesch, M. Jović, E. Villani, M. Sentic, S. Rapino, S. Arbault, F. Paolucci and N. Sojic, Single Cell Electrochemiluminescence Imaging: From the Proof-of-Concept to Disposable Device-Based Analysis, J. Am. Chem. Soc., 2017, 139, 16830-16837.

24 S. Pan, J. Liu and C. M. Hill, Observation of Local Redox Events at Individual $\mathrm{Au}$ Nanoparticles Using Electrogenerated Chemiluminescence Microscopy, J. Phys. Chem. C, 2015, 119, 27095-27103.

25 A. J. Wilson, K. Marchuk and K. A. Willets, Imaging Electrogenerated Chemiluminescence at Single Gold Nanowire Electrodes, Nano Lett., 2015, 15, 6110-6115.

26 M. J. Zhu, J. B. Pan, Z. Q. Wu, X. Y. Gao, W. Zhao, X. H. Xia, J. J. $\mathrm{Xu}$ and $\mathrm{H}$. $\mathrm{Y}$. Chen, Electrogenerated Chemiluminescence Imaging of Electrocatalysis at a Single Au-Pt Janus Nanoparticle, Angew. Chem., Int. Ed., 2018, 57, 1-6.

27 L. Qian and X. R. Yang, One-Step Synthesis of $\mathrm{Ru}\left(2,2^{\prime}\right.$ Bipyridine)3cl2-Immobilized Silica Nanoparticles for Use in Electrogenerated Chemiluminescence Detection, Adv. Funct. Mater., 2007, 17, 1353-1358. 
28 L. H. Zhang and S. J. Dong, Electrogenerated Chemiluminescence Sensors Using $\mathrm{Ru}(\mathrm{Bpy})_{(3)^{(2+)}}$ Doped in Silica Nanoparticles, Anal. Chem., 2006, 78, 5119-5123.

29 S. Zanarini, E. Rampazzo, L. D. Ciana, M. Marcaccio, E. Marzocchi, M. Montalti, F. Paolucci and L. Prodi, $\mathrm{Ru}(\mathrm{Bpy})_{3}$ Covalently Doped Silica Nanoparticles as Multicenter Tunable ftructures for Electrochemiluminescence Amplification, J. Am. Chem. Soc., 2009, 131, 2260-2267.

30 W. Miao, J. P. Choi and A. J. Bard, Electrogenerated Chemiluminescence 69: The Tris(2,2'-Bipyridine) Ruthenium(II), $\quad\left(\mathrm{Ru}(\mathrm{Bpy})_{3}{ }^{(2+)}\right) /$ Tri-N-Propylamine (Tpra) System Revisited-a New Route Involving Tpra*+ Cation Radicals, J. Am. Chem. Soc., 2002, 124, 14478-14485.

31 Y. Zu and A. J. Bard, Electrogenerated Chemiluminescence. 66. The Role of Direct Coreactant Oxidation in the Ruthenium Tris(2,2')Bipyridyl/Tripropylamine System and the Effect of Halide Ions on the Emission Intensity, Anal. Chem., 2000, 72, 3223-3232.

$32 \mathrm{Z}$. F. Chen and Y. B. Zu, Electrogenerated Chemiluminescence of the Tris(2,2'-Bipyridine) Ruthenium(II)/Tri-N-Propylamine (Tpra) System: Crucial Role of the Long Lifetime of Tpra(Center Dot+) Cation
Radicals Suggested by Electrode Surface Effects, J. Phys. Chem. C, 2008, 112, 16663-16667.

33 K. Imai, G. Valenti, E. Villani, S. Rapino, E. Rampazzo, M. Marcaccio, L. Prodi and F. Paolucci, Numerical Simulation of Doped Silica Nanoparticle Electrochemiluminescence, J. Phys. Chem. C, 2015, 119, 26111-26118.

34 M. Sentic, M. Milutinovic, F. Kanoufi, D. Manojlovic, S. Arbault and N. Sojic, Mapping Electrogenerated Chemiluminescence Reactivity in Space: Mechanistic Insight into Model Systems Used in Immunoassays, Chem. Sci., 2014, 5, 2568-2572.

35 S. J. Kwon, H. Zhou, F. R. F. Fan, V. Vorobyev, B. Zhang and A. J. Bard, Stochastic Electrochemistry with Electrocatalytic Nanoparticles at Inert Ultramicroelectrodes-Theory and Experiments, Phys. Chem. Chem. Phys., 2011, 13, 5394-5402. 36 Z. Chen, J. Li, X. Chen, J. Cao, J. Zhang, Q. Min and J. J. Zhu, Single Gold@Silver Nanoprobes for Real-Time Tracing the Entire Autophagy Process at Single-Cell Level, J. Am. Chem. Soc., 2015, 137, 1903-1908.

37 C. Ma, W. Wu, Y. Peng, M. X. Wang, G. Chen, Z. Chen and J. J. Zhu, A Spectral Shift-Based Electrochemiluminescence Sensor for Hydrogen Sulphide, Anal. Chem., 2018, 90, 1334-1339. 